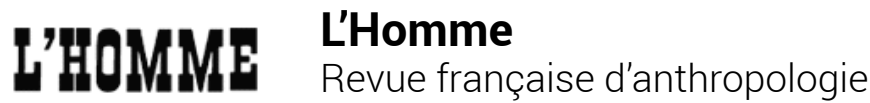

166 | avril-juin 2003

Malinowski, Faulkner. Culture et cognition. Souvenir et héritage

\section{Ana Mariella Bacigalupo, La Voz del Kultrun en la modernidad. Tradición y cambio en la terapéutica de siete machi mapuche}

Santiago, Ediciones Universidad Católica de Chile, 2001, 273 p., bibl., ill.

\section{Guillaume Boccara}

\section{(2) OpenEdition}

Journals

Édition électronique

URL : http://journals.openedition.org/lhomme/18713

DOI : 10.4000//homme.18713

ISSN : 1953-8103

Éditeur

Éditions de l'EHESS

Édition imprimée

Date de publication : 30 juin 2003

Pagination : 263-266

ISBN : 2-7132-1805-5

ISSN : 0439-4216

Référence électronique

Guillaume Boccara, «Ana Mariella Bacigalupo, La Voz del Kultrun en la modernidad. Tradición y cambio en la terapéutica de siete machi mapuche », L'Homme [En ligne], 166 | avril-juin 2003, mis en ligne le 08 septembre 2008, consulté le 21 septembre 2020. URL : http://journals.openedition.org//homme/ 18713 ; DOI : https://doi.org/10.4000//homme.18713

Ce document a été généré automatiquement le 21 septembre 2020.

(c) École des hautes études en sciences sociales 


\section{Ana Mariella Bacigalupo, La Voz del Kultrun en la modernidad. Tradición y cambio en la terapéutica de siete machi mapuche}

Santiago, Ediciones Universidad Católica de Chile, 2001, 273 p., bibl., ill.

\section{Guillaume Boccara}

1 LE LIVRE que consacre Ana Mariella Bacigalupo à l'étude du chamanisme mapuche a un objectif clair : montrer que les chamanes mapuche en particulier, et la culture qu'ils modèlent et qui les modèle en général, sont fondamentalement dynamiques et capables de faire face aux multiples agressions du monde moderne. Les chamanes (machi en mapudungun) ne sont pas les représentants d'une société mapuche primitive ou traditionnelle qui aurait succombé aux pressions acculturantes de la modernité néolibérale, étatique et nationale chilienne. Ils, ou plutôt elles (la grande majorité des machis sont en effet des femmes), jouent un rôle décisif dans la réponse indienne aux défis sociaux, psychologiques et culturels auxquels les Mapuche ont dû faire face depuis leur défaite militaire à la fin $\mathrm{du} \mathrm{xIx}^{\mathrm{e}}$ siècle. L'auteure observe que non seulement le chamanisme - système religieux jugé archaïque - ne disparaît pas sous l'influence déstructurante du monde moderne, mais que « la demanda por los servicios médicos y religiosos tradicionales de las machi se ha incrementado precisamente en las zonas cercanas a las ciudades donde el efecto de la modernización occidental es más fuerte » (p.10). Il s'agit donc, pour cette anthropologue d'origine chilienne qui enseigne aux États-Unis, de rendre compte de « este fenomeno de tradición, adaptación y cambio del rol de machi en la actualidad» (p. 11). Un problème anthropologique classique (analyser les changements et les permanences) que l'auteure se propose de résoudre à travers la comparaison des trajectoires de vie, pratiques et représentations de plusieurs chamanes dans le but d'échapper à l'illusion d'une culture mapuche homogène ou «typique », et en considérant la diversité des expériences chamaniques et du rôle des individus dans la production culturelle. 
2 La Voz del Kultrun en la Modernidad comporte deux parties. La première, consacrée à la présentation d'ensemble de la « culture mapuche », a pour but de fournir le background ethnographique minimum, nécessaire à la compréhension des cas de machis présentés en seconde partie. Dans les cent vingt premières pages, sont abordés, en des termes généraux, la vision du monde, le chamanisme, la conception de la maladie, les différents types de cures chamaniques et le nouveau rôle des chamanes dans un contexte caractérisé par l'hégémonie wingka (non mapuche). L'auteure résume ce qui a été dit et écrit au cours des quarante dernières années au sujet du dualisme mapuche (Louis Faron, Maria Ester Grebe), de la classification des maladies (Luca Citarella, Ivonne Jelves, Ana Maria Oyarce) ou encore de la domination exercée par la société chilienne (Guillaume Boccara, Wilson Cantoni, Roger Kellner, Milan Stuchlik). Cette synthèse est cependant décevante. Le contexte social est absent d'une partie qui se veut pourtant ethnographique. Nous ne savons pas ce que représentent ces comunidades sur lesquelles l'auteure insiste fort justement puisque «las machi ayudan a mantener la cohesión de la comunidad y su identidad frente a lo foráneo a través del respeto por las tradiciones » (p.22). Rien sur les unités territoriales, les groupes sociaux, le groupe domestique, ni sur les activités productives. Les relations de domination sociale, d'exploitation économique et d'assujettissement politique sont à peine évoquées, alors que Bacigalupo considère à juste titre que celles-ci déterminent en grande partie le contenu et la nature des pratiques et représentations chamaniques. Au reste, les auteurs ayant traité ces problèmes en détails (José Bengoa, Rodrigo Valenzuela, Cantoni, Kellner, A. Saavedra, Stuchlik) sont absents de la bibliographie.

3 Au sujet de la classification des maladies, des différents types de cures chamaniques et de la combinaison des systèmes médicaux en Araucanie, l'auteure offre une présentation appauvrie de l'ouvrage collectif publié en 1995, Medicinas y Culturas en la Araucanía (Santiago, Editorial Sud-Americana). De plus, elle est particulièrement laconique sur deux thèmes: la conception de la personne et la conceptualisation de l'environnement; thèmes pourtant inévitables pour qui se propose d'étudier le chamanisme en tant que système visant à l'interprétation, à la prévention et au traitement du mal, de la maladie, de l'infortune ou des catastrophes « naturelles ». À ce propos, il aurait été intéressant de montrer la richesse sémantique des termes en mapudungun. Qui plus est, l'étude de l'usage discriminant que font les Mapuche des deux langues (espagnol et mapudungun) aurait été des plus instructives car il varie en fonction du contexte, des thèmes abordés, de l'état psychique, etc. Leur capacité à passer d'une langue à l'autre, en opérant de véritables traductions culturelles, nous aurait certainement appris beaucoup sur la superposition de deux systèmes symboliques ou sur l'incorporation d'éléments exogènes à l'univers mental indigène.

4 Je partage l'opinion de Bacigalupo selon laquelle les chamanes sont des agents médiateurs. Mais encore s'agit-il de la démontrer en restituant les structures symboliques et les mécanismes complexes d'incorporation, d'emprunt, de fusion ou de superposition. Les récents travaux de José Quidel et Juan Carlos Gumucio sur le notion de che (personne) et ceux d'ethnobotanistes mapuche auraient dû être cités. La nonutilisation de textes en langue vernaculaire et l'absence de réflexion sur le passage de l'espagnol au mapudungun expliquent le manque d'attention portée, dans le discours mapuche, sur ce que l'auteure appelle la modernidad. On connait pourtant l'importance de la parole et du chant chez les Mapuche (Mischa Titiev) et l'on sait que c'est à travers l'usage de figures rituelles et d'entités intermédiaires entre ce monde-ci et le monde- 
autre (witranalwe, anchimallen, piwichen, sumpall, meulen, etc.) que ces Indiens donnent leur point de vue sur l'histoire, la politique, l'économique, les relations de travail (Boccara, Rolf Foerster). On quitte cette première partie avec une idée simplifiée, ou consensuelle, de la culture mapuche typique, pour entrer alors dans la seconde où cet édifice culturel va s'effriter page après page. L'auteure explique tout d'abord qu'un Mapuche ne consulte que rarement la machi de sa propre communauté, lui préférant celles des communautés proches ou plus lointaines (pp. 214, 223). On apprend que la plupart des machis sont aussi des kalku (sorcières). Mais le problème du rôle des chamanes dans les dynamiques sociales intra-, inter-communautaires et interethniques peut-il être résolu si nous manquons de données sociohistoriques de base concernant les communautés en question? Comment prendre la mesure du rôle social et politique des machis et de l'importance de la vengeance au sein de la société mapuche si nous ne savons presque rien

des relations sociales au sein des groupes étudiés. Les chamanes affirment pourtant eux-mêmes que «la información sobre la familia del paciente, las relaciones sociales, los incidentes que preceden la enfermedad y las relaciones con los vecinos son cruciales para diagnosticar las enfermedades y sanar el paciente" (pp. 232-233). Comment évaluer la typologie dressée par l'auteure entre différents types de chamanes si nous ne savons rien de leurs chants et rythmes (seuls deux courts extraits de machi ül sont retranscrits en espagnol, pp.177, 181), des plantes qu'elles utilisent (aucune étude ethnobotanique citée) et de leurs techniques de cure (description extrêmement sommaire des principales cures). Une attention plus soutenue sur la catégorie de newen (pouvoir) aurait certainement permis à l'auteure de s'apercevoir que lorsqu'une machi perd en pouvoir d'un côté, une autre machi gagne en pouvoir de l'autre. Bref, l'absence d'usage du mapudungun et de tout contexte historique, social et économique donne à cette seconde partie des airs de telenovela (pp. 202, 218). En l'absence d'ethnographie minutieuse, on ne s'étonnera donc pas de l'impossibilité de comparer si ce n'est des traits isolés entre eux. À partir des éléments qui nous sont fournis dans ce livre, nous ne pouvons pas juger de la validité de l'hypothèse d'une supposée spécialisation récente des différents thérapeutes (pp. 111-113), tout comme nous ne pouvons rien dire de la typologie dressée par Bacigalupo, sinon qu'elle est bien «traditionnelle » dans la mesure où les machis « traditionnelles » sont distinguées des machis plus " modernes » ou adaptadas (p. 265). L'auteure reprend ainsi la vieille dichotomie tradition/modernité (p. 239), rejetée à juste titre en introduction (p.9), ce qui la conduit à donner des réponses peu satisfaisantes à des problèmes mal posés: "Aunque Rocio es una machi tradicional, ella acepta la tecnología moderna » (p.150). Si l'on apprend que l'auteure croit (tiene fe) aux machis et à leurs pouvoirs curatifs (elle a été soignée par la plupart des chamanes mentionnés dans le livre), en revanche on ne sait rien de la signification de la notion de fe pour les Mapuche et de ce qui détermine les fluctuations de la foi. Il semble pourtant que l'auteure tenait là un élément essentiel qui lui aurait permis d'analyser les rapports entre culture, société et pouvoir puisque comme elle l'écrit ellemême : «El elemento más importante en el poder y el prestigio de una machi es la fe » (p. 268). Si l'on décrypte cette formule un peu confuse, il semblerait que l'auteure se réfère à ce que l'on nomme ordinairement le pouvoir symbolique.

5 Signalons pour finir quelques erreurs et manques. Contrairement à ce qu'écrit Bacigalupo, il n'existe pas de matrilignage dans la société mapuche (p. 155) ; la particule tun ne signifie pas cérémonie mais indique une action (p. 87); le wetripantu n'est pas le Nouvel An mapuche mais la nouvelle sortie (we-tripan) du soleil (antü) célébrée depuis 
plusieurs décennies sous le nom de San Juan et correspondant au solstice d'hiver; il n'existe pas de race mapuche (p. 266), même s'il convient de préciser que pour ces Indiens le sang (mollfüñ) est un marqueur d'ethnicité (mais, de cela, l'auteure ne parle pas) ; konpapüllü ne signifie pas con espíritu (p. 84) mais de façon plus intéressante que le püllü (une des multiples composantes du che) est venu (pa) et a pénétré (konün) la personne ; enfin le mudai est une boisson fermentée à base de blé ou de maïs (pp. 142, 155, 241). Pour continuer dans ce registre, on observera que pour les Mapuche le cœur (piuke) et la pensée (rakiduam) sont deux composantes fondamentales de la personne mais elles ne sont que brièvement évoquées par Bacigalupo. Aucune analyse socioethnologique des principaux rites d'initiation (machiluwün) et de rénovation de pouvoir (ngeikurewen) n'est proposée, tout comme est absente une réflexion sur la valeur de la monnaie (pourtant cruciale pour l'efficacité des cures). Les vêtements jouent effectivement le rôle de marqueur ethnique mais il est surprenant que Bacigalupo, qui s'intéresse aux relations entre genre et ethnicité, ne signale pas que ce qui distingue une femme mapuche d'une chiñura (femme chilienne ou señora) est la façon d'attacher le foulard couvrant ses cheveux (les Mapuche nouent leur pañuelo sur le devant). Enfin, l'auteure n'entreprend aucune étude de cas ou de trajectoire thérapeutique, ne fait qu'effleurer les effets de la pénétration pentecôtiste et ne discute pas le nouveau rôle des machis dans la politique extérieure mapuche et dans la renaissance indienne. Bref, voici un ouvrage assez décevant, dont on ne perçoit pas très bien comment il pourra changer les préjugés de la mayoría chilena (p. 9) à l'égard des machis et des Mapuche.

\section{AUTEUR}

GUILLAUME BOCCARA

Yale University, New Haven, Connecticut. 